conjugates of $\alpha$ is in $\mathfrak{B}_{i}, i=1, \cdots, m$; hence neither is any power of their product. Some such power, however, is in $\Re$, hence in $\mathfrak{B} \cap \Re \subset \mathfrak{P}_{1}$. This is a contradiction and completes the proof.

\author{
HARVARD UNIVERSITY AND \\ Massachusetts Institute of TechNology
}

\title{
NOTE ON AN ASYMMETRIC DIOPHANTINE APPROXIMATION
}

\author{
C. D. OLDS
}

1. Introduction. In a recent paper B. Segre [1] ${ }^{1}$ introduced a new type of Diophantine approximation which he called asymmetric, since the intervals of approximation are divided into two partial intervals which are in an arbitrarily given ratio. His main result is the following theorem $[1$, p. 357$]$ :

THEOREM 1. Every irrational $\theta$ has an infinity of rational approximations $x / y$ such that

$$
\frac{-1}{y^{2}(1+4 \tau)^{1 / 2}}<\frac{x}{y}-\theta<\frac{\tau}{y^{2}(1+4 \tau)^{1 / 2}} \quad(y>0),
$$

where $\tau$ is any given non-negative real number.

This theorem is classic for $\tau=0$, cf. [2, p. 139], and for $\tau=1$ it reduces to the fundamental result due to Hurwitz [2, p. 163]. No other particular cases of the theorem seem to be known.

Segre's proof of (1) is geometrical. The purpose of this note is to show that when $\tau \geqq 1$ it is possible to give a very simple arithmetical proof. The method is a generalization of that used by Khintchine [3] for the special case when $\tau=1$.

2. Proof of Theorem 1. We suppose that $\theta$ is irrational and that $0<\theta<1$. For an arbitrary positive integer $n$ form the Farey series ${ }^{2}$ of order $n$, that is, the ascending series of irreducible fractions between 0 and 1 whose denominators do not exceed $n$. Let $a / b$ and $a^{\prime} / b^{\prime}$ be the two successive terms of this series which satisfy the inequalities $a / b<\theta<a^{\prime} / b^{\prime}$. We distinguish two cases.

Received by the editors October 4, 1945.

${ }^{1}$ Numbers in brackets refer to the references.

${ }^{2}$ See Hardy and Wright [2, p. 23]. 
Case 1. Suppose that $\tau>0$, and that $b / b^{\prime}>(\xi+1) / 2 \tau$, or $b / b^{\prime}$ $<(\xi-1) / 2 \tau$, where $\xi=(1+4 \tau)^{1 / 2}$. Then setting $\omega=b / b^{\prime}$ we see that

$$
\frac{1}{\xi}\left(\tau+\frac{1}{\omega^{2}}\right)-\frac{1}{\omega}=\frac{\tau}{\xi \omega^{2}}\left(\omega-\frac{\xi+1}{2 \tau}\right)\left(\omega-\frac{\xi-1}{2 \tau}\right)>0 ;
$$

consequently, since $a^{\prime} b-a b^{\prime}=1$,

$$
\frac{a^{\prime}}{b^{\prime}}-\frac{a}{b}=\frac{1}{b^{\prime 2} \omega}<\frac{1}{b^{\prime 2} \xi}\left(\tau+\frac{1}{\omega^{2}}\right)=\frac{\tau}{b^{\prime 2 \xi}}+\frac{1}{b^{2} \xi},
$$

which implies that

$$
\frac{a}{b}+\frac{1}{b^{2} \xi}>\frac{a^{\prime}}{b^{\prime}}-\frac{\tau}{b^{\prime 2} \xi}
$$

Hence $\theta$ must be interior to one or the other of the intervals

$$
\left(\frac{a}{b}, \frac{a}{b}+\frac{1}{b^{2} \xi}\right) \text { or }\left(\frac{a^{\prime}}{b^{\prime}}-\frac{\tau}{b^{\prime 2} \xi}, \frac{a^{\prime}}{b^{\prime}}\right) \text {. }
$$

Then, according as $\theta$ belongs to the first or to the second interval, we have, respectively, the inequalities

$$
-1 / b^{2} \xi<a / b-\theta<0, \text { or } 0<a^{\prime} / b^{\prime}-\theta<\tau / b^{\prime 2} \xi \text {. }
$$

Thus (1) is true, where for $y$ we take either $b$ or $b^{\prime}$. The infinity of solutions is assured since, for irrational $\theta, b$ and $b^{\prime}$ increase as $n$ increases.

If $\omega=b / b^{\prime}=(\xi \pm 1) / 2 \tau$, then both $\tau$ and $\xi$ must be rational. Then the inequality sign in (4) is replaced by an equality sign, and the right and left end points of the intervals in (5) coincide. But $\theta$, being irrational, cannot be equal to this common end point. Hence $\theta$ must be interior to one or the other of these intervals, and the proof proceeds as already explained.

Case 2. We now suppose that $(\xi-1) / 2 \tau<b / b^{\prime}<(\xi+1) / 2 \tau$. We consider in turn the two sub-intervals

$$
\left(\frac{a}{b}, \frac{a+a^{\prime}}{b+b^{\prime}}\right) \text { and }\left(\frac{a+a^{\prime}}{b+b^{\prime}}, \frac{a^{\prime}}{b^{\prime}}\right) .
$$

For the first sub-interval, write $\omega=b /\left(b+b^{\prime}\right)$. Then it is easy to see that $\omega<(\xi-1) / 2 \tau, \tau>0$, and that the inequality (2) is again true. Hence

$$
\frac{a+a^{\prime}}{b+b^{\prime}}-\frac{a}{b}=\frac{1}{\left(b+b^{\prime}\right)^{2} \omega}<\frac{1}{b^{2} \xi}+\frac{\tau}{\left(b+b^{\prime}\right)^{2 \xi}},
$$


and, proceeding as in Case 1, we see that (1) is true where for $y$ we take either $b$ or $b+b^{\prime}$.

The second sub-interval is handled in exactly the same way. We set $\omega=\left(b+b^{\prime}\right) / b^{\prime}$, then $\omega>(\xi+1) / 2 \tau$, provided $\tau \geqq 1$. This is the first time we need this restriction on $\tau$. The inequality (1) is again true where $y$ is either $b^{\prime}$ or $b+b^{\prime}$.

\section{REFERENCES}

$1 \mathrm{~B}$. Segre, Lattice points in infinite domains, and asymmetric Diophantine approximations, Duke Math. J. vol. 12 (1945) pp. 337-365.

2. G. H. Hardy and E. M. Wright, An introduction to the theory of numbers, Oxford, 1938.

3. A. Khintchine, Neuer Beweis und Verallgemeinerung eines Hurwitzschen Satzes, Math. Ann. vol. 111 (1935) pp. 631-637.

San Jose State College 